\title{
SCHWANNOMA CUTÂNEO EM CÃO BOXER - RELATO DE CASO
}

\author{
RAMOS, Lígia Marina Piai Dias ${ }^{1}$; \\ DANEZE, Edmilson Rodrigo'; \\ MAGALHÃES, Geórgia Modé; \\ OLIVEIRA, Silvana Alvarez de $\mathrm{d}^{4}$;
}

\footnotetext{
${ }^{1}$ Acadêmica do Curso de Medicina Veterinária, Faculdade Dr. Francisco Maeda (FAFRAM/FE), ${ }^{2}$ Médico Veterinário, Programa de Aprimoramento em Clínica e Cirurgia Veterinária, Faculdade Dr. Francisco Maeda (FAFRAM/FE), ), 3 Médica Veterinária, Professora Doutora, Faculdade Dr. Francisco Maeda (FAFRAM/FE), ),

${ }^{4}$ Médica Veterinária Autônoma.
}

\section{RESUMO}

S chwannoma refere-se à neoplasia que se origina das células de Schwann, localizadas no sistema nervoso periférico e são incomuns em cães. Um cão, macho, da raça Boxer, com dez anos de idade, foi encaminhado com queixa principal de um nódulo cutâneo em localização torácica lateral direita, próximo às primeiras costelas. Durante o exame clínico observou-se que o nódulo media aproximadamente $5 \mathrm{~cm}$ de diâmetro, possuindo formato arredondado, consistência firme e não ulcerado. Foram realizados exames préoperatórios de rotina e optou-se pela retirada cirúrgica da massa. Na microscopia de luz observou-se uma massa de característica neoplásica apresentando formato ovalado bem delimitada e com crescimento expansivo. As células estavam distribuídas em ninhos com feixes celulares. Os núcleos eram fusiformes e de tamanhos variados. Não foram observadas figuras de mitoses. O diagnóstico foi baseado nas descrições morfológicas das células neoplásicas. Conclui-se que o Schwannoma foi uma neoplasia de características histológicas e comportamento benigno devido à localização distal a nervos da raiz da medula.

Palavras-chave: Canino. Neoplasia. Sistema nervoso. 


\section{INTRODUÇÃO}

A pele e o tecido subcutâneo são os locais mais frequentemente acometidos por neoplasias em cães. Essas lesões podem ter origem ectodérmica, mesodérmica ou melanocítica, sendo as neoplasias de origem ectodérmica mais comuns (ZACHARY, 2009).

O sistema nervoso é dividido em sistema nervoso central (SNC) e sistema nervoso periférico (SNP) (SWENSON; REECE, 2006). No SNP todos os axônios estão envolvidos por células especializadas, denominadas células de Schwann, que Ihes fornecem suporte estrutural e metabólico. No SNC, a mielinização é semelhante à do SNP, exceto pelo fato de que as bainhas de mielina são formadas por células denominadas oligodentrócitos (GARTNER; HIATT, 2003; JUNQUEIRA; CARNEIRO, 2004).

Em humanos, as neoplasias de nervos periféricos são as mais comuns e representam cerca de $8 \%$ das neoplasias intracranianas e $29 \%$ das neoplasias primárias de medula espinhal (WOODRUFF et al., 2000; ZAMECNIK; MICHAL, 2001). No entanto, tais neoplasias não são comuns em cães, apresentando incidência de 0,5\% (GROSS et al., 2009) e representando $27 \%$ de todas as neoplasias do sistema nervoso de cães (COSTA, 2009).

O termo schwannoma é utilizado quando as células neoplásicas se originam apenas das células de Schwann (GOLDSCHMIDT; HENDRICK, 2002; GROSS et al., 1992; SCOTT et al., 2001) ou quando há clara associação delas com os troncos nervosos (YAGER; SCOTT, 1993; YAGER; WILCOCK, 1994), já o termo neurofibroma é aplicado quando a neoplasia é composta de células de Schwann e células perineurais (GOLDSCHMIDT; HENDRICK, 2002).

O schwannoma é uma neoplasia primitiva dos nervos, encapsulada, geralmente solitária e raramente múltipla; localiza-se nas raízes dos nervos cranianos e espinhais, sendo ocasionalmente encontrada no trajeto periférico dos nervos (DUARTE et al., 1994). Embora a recorrência local possa ser decorrente de uma ressecção incompleta (GIROLAMI et al., 2005), a maioria é benigna, mas existem casos malignos (MCCLENATHAN; BLOOM, 2004; MIURA et al., 2003; RODRIGUEZ et al., 2004) e, a menos que sua localização interfira nas funções vitais, a neoplasia benigna não causa a morte (PORTH, 2004). 
Nesse contexto, o presente trabalho tem como objetivo relatar um caso de schwannoma em tecido cutâneo de um cão Boxer, tendo em vista a baixa incidência dessa afecção na Medicina Veterinária.

\section{RELATO DE CASO}

Um cão, macho, da raça Boxer, com dez anos de idade, foi atendido na clínica veterinária apresentando, como queixa principal, um nódulo cutâneo em localização torácica lateral direita, próximo às primeiras costelas.

Durante o exame clínico observou-se que o nódulo media aproximadamente $5 \mathrm{~cm}$ de diâmetro, possuindo formato arredondado, consistência firme e não ulcerado. Não foram visualizadas alterações significativas nos exames de imagem realizados (radiografia torácica abrangendo o membro direito e ultrassonografia abdominal), e os resultados dos exames hematológicos (hemograma e bioquímica sérica) encontravam-se dentro dos padrões de normalidade. Assim, o tratamento indicado foi a exérese cirúrgica, realizado com base nos princípios da cirurgia oncológica, ou seja, exérese cirúrgica completa mantendo-se margem livre de pelo menos $3 \mathrm{~cm}$ a partir da cápsula neoplásica em todas as direções, retirando o tumor em bloco sem incidir o seu interior ou a cápsula tumoral. O nódulo foi acondicionado em frasco contendo solução de formalina a $10 \%$ e encaminhado para realização de exame histopatológico.

\section{RESULTADOS E DISCUSSÃO}

Na microscopia de luz observou-se tecido com ausência de epiderme e derme, sendo somente a região subcutânea (tecido adiposo) reconhecida no local. Observou-se, ainda, tecido de característica neoplásica apresentando formato ovalado bem delimitado e com crescimento expansivo, além de algumas áreas de necrose. As margens cirúrgicas estavam livres da neoplasia. As células estavam distribuídas em ninhos com feixes celulares. Os núcleos eram fusiformes e de tamanhos variados. O citoplasma era eosinofílico com bordos não bem definidos. Não foram observadas figuras de mitoses. O diagnóstico foi baseado nas 
características morfológicas da neoplasia, definindo como schwannoma ou neurofibroma (Figuras 1 e 2).

Após 12 meses do procedimento cirúrgico o animal não apresentou nenhuma alteração em seu estilo de vida e comportamento, assim como não foram observadas recidivas macroscópicas da neoplasia.

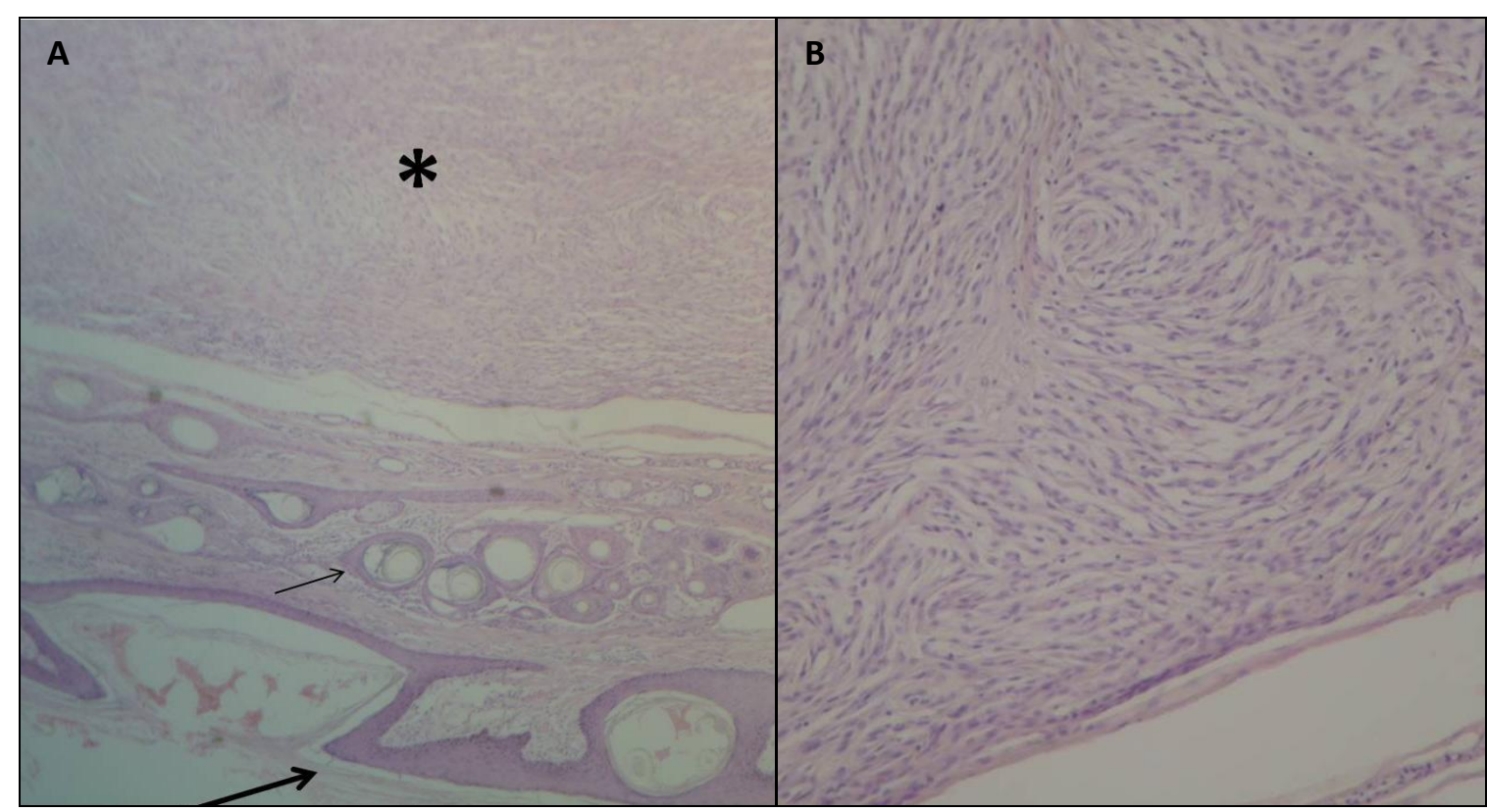

Figura 1. Schwannoma cutâneo, cão, Boxer, 10 anos de idade. Em A, notar epiderme (seta maior) e anexos cutâneos (seta menor) e neoplasia benigna de nervos periféricos ( $\left.{ }^{*}\right), \mathrm{HE}$, aumento de 40x. Em B, notar moderada celularidade e pouca matriz celular, e células fusiformes em padrão de feixes e ninhos, HE, aumento de $100 x$. 


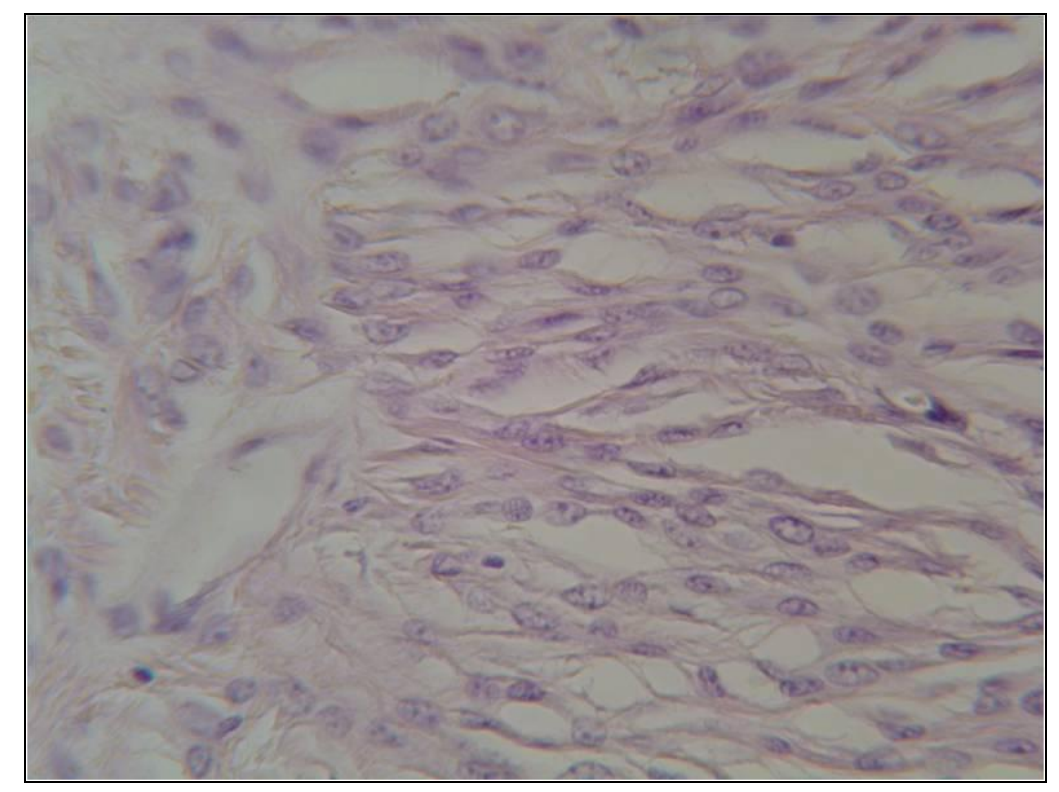

Figura 2. Schwannoma cutâneo, cão, Boxer, 10 anos de idade. Notar moderado pleomorfismo celular, as células encontram-se alongadas e os núcleos ovais regulares. HE, aumento de 400x.

Os schwannomas são mais comuns em cães entre cinco e 12 anos, com idade média de nove anos para o surgimento da neoplasia (GROSS et al., 2009; WOODRUFF et al., 2000), sendo incomum em animais de menos de três anos de idade. Neste trabalho, o animal relatado era macho, da raça Boxer e apresentava 10 anos de idade, ou seja, ele enquadrava-se dentro da faixa etária de maior incidência. No entanto, não há relatos sobre predisposição racial para a afeç̧ão (WOODRUFF et al., 2000). Costa (2009) refere que cães de grande porte parecem ser mais comumente afetados, enquanto que Santos e Alessi (2010) citam que a mesma ocorre com maior frequência em fêmeas.

A localização do nódulo relatado concorda com a literatura, sendo a mais comum próxima ao plexo braquial. As localizações mais citadas são entre as raízes nervosas de C6 a T2 e nervos periféricos do plexo braquial (ARAÚJO et al., 2008; CHRISMAN et al., 2005; SEIM, 2005; SHELL; INZANNA, 2003; SHORES, 1996). O local primário de origem pode estar em qualquer raiz nervosa espinhal, ramo ventral ou nervo periférico.

Devido a sua baixa incidência, tal neoplasia é pouco diagnosticada, podendo ser um achado incidental de exame ultrassonográfico ou de tomografia computadorizada (TERRA JÚNIOR et al., 2012). No entanto, para a diferenciação dos schwannomas em relação a outras 
alterações, podem ser feitos exames citopatológicos (KOESTNER; HIGGINS, 2002). Contudo, o diagnóstico definitivo é baseado nos achados histopatológicos, em que uma variedade de alterações degenerativas pode ser encontrada nos schwannomas, incluindo pleomorfismo nuclear, alteração xantomatosa e hialinização celular.

A imuno-histoquímica por meio da proteína S-100 também é um dos recursos utilizados na identificação dos schwannomas (ERDOGAN et al., 2004; GIROLAMI et al., 2005; HENN et al., 1998; MESSERLI et al., 2002; RODRIGUEZ et al., 2004; TERRA JÚNIOR et al., 2012); contudo, a morfologia das células caracterizam bem a neoplasia.

O tecido conjuntivo é mais proeminente nos neurofibromas do que nos schwannomas. Essa diferença na quantidade de tecido conjuntivo expresso em cada neoplasia pode ser explicada pelos componentes celulares que formam as mesmas. Os schwannomas são compostos unicamente por células de Schwann ao contrário dos neurofibromas que são formados por uma grande variedade de tipos celulares incluindo fibroblastos e células perineurais (HIROSE et al., 1998). A presença e, principalmente, a quantidade de tecido conjuntivo podem auxiliar na diferenciação entre esses dois tipos de neoplasias, no presente caso, observou-se pouco tecido conjuntivo e apenas um tipo celular presente no tecido neoplásico observado ao microscópio, caracterizando-o como schwannoma.

Quanto ao aspecto macroscópico, os schwannomas são arredondados, sólidos, bem delimitados e crescem comprimindo e nunca invadindo (SANTOS; ALESSI, 2010). Apresentam-se bem circunscritos e, na maioria dos casos, estão ligados ao nervo, mas podem ser separados dele. Tais neoplasias formam massas acinzentadas firmes, porém podem também apresentar áreas de alterações císticas e xantomatosas (GIROLAMI et al., 2005). Caracterizam-se por taxa lenta e progressiva de crescimento, podendo se manter ou regredir. São envolvidos por uma cápsula fibrosa que é responsável por uma linha que possibilita visualizar uma demarcação entre a neoplasia e os tecidos adjacentes facilitando assim a remoção cirúrgica. Acredita-se que essa cápsula é a representação da reação dos tecidos vizinhos a lesão, diferente das neoplasias malignas (PORTH, 2004).

No exame microscópico, os schwannomas mostram uma mistura de dois padrões de crescimento: o padrão Antoni A, células alongadas com expansões citoplasmáticas 
arranjadas em fascículos nas áreas de celularidade moderada à alta com pouca matriz estromal; podendo apresentar zonas livres de núcleos, que se situam entre as regiões de paliçadas nucleares irregulares intercalados com abundante colágeno e que são denominadas corpos de Verocay, e o padrão Antoni B, no qual a neoplasia é menos densamente celular com um mesclado frouxo de células ao longo das alterações microcísticas e mixóides. Em ambas as áreas, a citologia das células individualizadas é similar, com forma celular alongada e núcleos ovais regulares (GIROLAMI et al., 2005). Tais informações condizem com o presente caso, em que foi observada uma neoplasia cujas características enquadravam-se no padrão Antoni A.

Devido ao crescimento lento e a origem mesenquimatosa, tais neoplasias são insensíveis à quimioterapia e radioterapia (SEIM, 2005). O tratamento indicado é cirúrgico, com a retirada da massa em bloco, sem incidir o seu interior ou a cápsula adjacente, a fim de evitar a disseminação de células neoplásicas para tecidos sadios, o que neste caso apresentou bom resultado, pois em 12 meses de observação não houve recidiva (COSTA, 2009; FLANDERS, 2004; SHELL; INZANA, 2003; THEILEN; MADEWELL, 1979).

Segundo Koestner e Higgins (2002), o resultado das intervenções cirúrgicas demonstrou que o prognóstico das neoplasias do SNP, particularmente dos schwannomas, é desfavorável. No entanto, as neoplasias localizadas distalmente da medula espinhal tem melhor prognóstico do que as neoplasias localizadas próximas aos nervos descendentes e dos plexos braquiais e lombossacro. No presente caso, a neoplasia era localizada no tecido cutâneo torácico, próximo às primeiras costelas, e distal a nervos da raiz da medula espinhal, ou seja, uma neoplasia de melhor prognóstico quando comparada com as demais.

\section{CONCLUSÃO}

Concluímos que, no presente caso, o schwannoma diagnosticado apresentava características histológicas benignas e o procedimento cirúrgico, realizado com margens de segurança, teve bom resultado, já que a localização da neoplasia era cutânea e distal a nervos da raiz da medula espinhal. 


\section{CUTANEOUS SCHWANNOMA IN A BOXER DOG - CASE REPORT}

\section{ABSTRACT}

S chwannoma refers to a neoplasia that originates from the Schwann cells, which are located in the peripheral nervous system, and is unusual in dogs. A male ten years old Boxer dog, was referred with the main complaint of a cutaneous nodule in the right lateral thoracic region, near the first rib. During the clinical examination it was observed that the nodule was round, firm and non-ulcerated and measured about $5 \mathrm{~cm}$ in diameter. Examinations were performed at the preoperative routine and we opted for surgical removal of the mass. The light microscopy showed neoplastic cells presenting oval features and expansive growth. The cells were distributed in nests with standard bundles. The nuclei were fusiform and had varying sizes. There were no mitosis figures. The diagnosis was based on the morphological descriptions of the neoplastic cells. We conclude that the schwannoma had histological features and benign behavior due to the distal spinal cord nerves location.

Keywords: Canine. Neoplasia. Nervous system.

\section{PIEL SCHWANNOMA EN PERRO BOXER - INFORME DE CASO}

\section{RESUMEN}

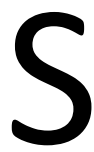
chwannoma se refiere a una neoplasia que se origina a partir de células de Schwann localizadas en el sistema nervioso periférico y que son inusuales en los perros. Un perro, macho de la raza Boxer, con diez años de edad, fue remitido con una queja principal de un nódulo cutáneo de localización torácica en el lado derecho, cerca de la primera costilla. En el examen clínico se observó que el nódulo medía aproximadamente 5 $\mathrm{cm}$ de diámetro, que tenía una forma redondeada, de consistencia firme y no ulcerada. Se realizaron pruebas de rutina preoperatorias y se optó por la extirpación quirúrgica de la masa. La microscopía óptica reveló una masa de características neoplásicas mostrando formato ovalado y bien definido, con crecimiento expansivo. Las células se organizaban en nidos con haces de células. Los núcleos mostraron una variación de tamaño moderado, siguiendo un formato fusiforme. No se observaron figuras mitóticas. El diagnóstico se basa en las descripciones morfológicas de las células neoplásicas, que caracteriza a un schwannoma. Se concluyó que el schwannoma era una neoplasia de características histológicas y comportamiento benigno debido a la ubicación distal a los nervios de la raíz de la médula espinal.

Palabras clave: Perro. Neoplasia. Sistema nervioso. 


\section{REFERÊNCIAS}

ARAÚJO, B. M.; KEMPER, B.; FIGUEIREDO, M. L.; CHIORATTO, R.; MARQUES, N. B.; TUDURY, E. A. Schwannoma do plexo braquial em cão. Medicina Veterinária, v. 2, n. 4, p. 45-49, 2008.

CHRISMAN, C.; MARIANI, C.; PLATT, S.; CLEMMONS, R. Monoparesia ou monoplegia. In: Neurologia para o clínico de pequenos animais. São Paulo: Roca, 2005. p. 303-314.

COSTA, R. C. Neoplasias do sistema nervoso. In: DALECK, C. R.; DE NARDI, A. B.; RODASKI, S. Oncologia em cães e gatos. São Paulo: Roca, 2009. p. 411-436.

DUARTE, F.; PITTELLA, J. E. H.; AVILA, C. M.; ROSEMBERG, S.; HAHN, M. D.; CHIMELLI, L.; DE PAOLA, D. Sistema nervoso. In: BRASILEIRO FILHO, G.; PITTELLA, J. E. H.; PEREIRA, F. E. L.; BAMBIRRA, E. A.; BARBOSA, A. J. A. Bogliolo Patologia. 5a ed. Rio de Janeiro: Guanabara Koogan, 1994. p. 723-825.

ERDOGAN, S.; TUNCER, U.; GUMURDULU, D.; ZORLUDEMIR, S.; KARAOGLAN, A.; YALINIZ, A.; ULUS, T. Primary peritracheal schwannoma: report of a case. Surgery Today, v. 34, p. 444446, 2004.

FLANDERS, J. A. Princípios de oncologia cirúrgica. In: ROSENTHAL, R. C. Segredos em oncologia veterinária. São Paulo: Artmed, 2004. p. 85-91.

GARTNER, L. P.; HIATT, J. L. Tratado de histologia. 2a ed. Rio de Janeiro: Guanabara Koogan, 2003. p. 158-161.

GIROLAMI, U.; ANTHONY, D. C.; FROSCH, M. P. O sistema nervoso central. In: KUMAR, V.; ABBAS, A. K.; FAUSTO, N. Robbins \& Cotran. Patologia. Bases patológicas das doenças. 7ạ ed. Rio de Janeiro: Elsevier, 2005. p. 1411-1486.

GOLDSCHMIDT, M. H.; HENDRICK, M. J. Tumors of the skin and soft tissues. In: MEUTEN, D. J. Tumors in domestic animals. 4a ed. Ames: lowa State, 2002. p. 44-117.

GROSS, T. L.; IHRKE, P. J.; WALDER, E. J. Veterinary dermatopathology: a macroscopic and microscopic evaluation of canine and feline skin disease. St. Louis: Mosby, 1992. 520p.

GROSS, T. L.; IHRKE, P. J.; WALDER, E. J.; AFFOLTER, V. K. Tumores neurais e perineurais. In: GROSS, T. L.; IHRKE, P. J.; WALDER, J. E.; AFFOLTER, K. V. Doenças de pele do cão e do gato. 2a ed. São Paulo: Roca, 2009. p. 770-780.

HENN, L. A.; GONZAGA, R. V.; CRESTANI, J.; CERSKI, M. R. Schwannoma intercostal simulando neoplasia pulmonar. Revista da Associação Médica Brasileira, v. 44, n. 2, p. 146-148, 1998. 
HIROSE, T.; SCHEITHAUER, B. W.; SANO, T. Perineurial malignant peripheral nerve sheath tumor (MPNST): A clinicophatologic, immunohistochemical and ultrastructural study of seven cases. American Journal of Surgical Pathology, v. 22, p. 1368-1378, 1998.

JUNQUEIRA, L. C.; CARNEIRO, J. Histologia básica: texto e atlas. 10a ed. Rio de Janeiro: Guanabara Koogan, 2004. p. 172-177.

KOESTNER, A.; HIGGINS, R. J. Tumors of the nervous system. In: MEUTEN, D. J. Tumors of domestic animals. 4a ed. lowa: lowa State, 2002. p. 697-738.

MCCLENATHAN, J. H.; BLOOM, R. J. Peripheral tumors of the intercostal nerves. Annals of Thoracic Surgery, v. 78, p. 713-714, 2004.

MESSERLI, S. M.; TANG, Y.; GIOVANNINI, M.; BRONSONX, R.; WEISSLEDER, R.; BREAKEFIELD, $X$. O. Detection of spontaneous schwannomas by MRI in a transgenic murine model of neurofibromatosis type 2. Neoplasia, v. 4, n. 6, p. 501-509, 2002.

MIURA, J.; DOITA, M.; MIYATA, K.; YOSHIYA, S.; KUROSAKA, M.; YAMAMOTO, H. Horner's Syndrome caused by a thoracic dumbbell-shaped schwannoma: sympathetic chain reconstruction after a one-stage removal of the tumor. Spine, v. 28, n. 2, p. E33-E36, 2003.

PORTH, C. M. Fisiopatologia. 6a ed. Rio de Janeiro: Guanabara Koogan, 2004. 1480p.

RODRIGUEZ, C. A.; MUNHOZ, A. H. N.; ZAMPIER, J. A.; SILVA, A. P. G.; FUSTES, O. H. Schwannoma benigno do nervo intercostal simulando neoplasia de pulmão. Arquivos de Neuropsiquiatria, v. 62, n. 4, p. 1100-1103, 2004.

SANTOS, R. L.; ALESSI, A. C. Patologia veterinária. São Paulo: Roca, 2010. 904p.

SCOTT, D. W.; MILLER, W. H.; GRIFFIN, C. E.; MULLER, K. Dermatologia de pequenos animais. 6a ed. Philadelphia: Saunders Company, 2001. 1528p.

SEIM, H. B. Cirurgia dos nervos periféricos. In: FOSSUM, T. W. Cirurgia de pequenos animais. 2a ed. São Paulo: Roca, 2005. p. 1336-1350.

SHELL, L. G.; INZANA, K. D. Neuropatias periféricas. In: BIRCHARD, S. J.; SHERDING, R. G. Manual Saunders: clínica de pequenos animais. São Paulo: Roca, 2003. p. 1421-1430.

SHORES, A. Moléstias traumáticas e neoplásicas do plexo braquial. In: BOJRAB, M. J. Mecanismos da moléstia na cirurgia de pequenos animais. São Paulo: Manole, 1996. p. 1353-1359.

SWENSON, M. J.; REECE, W. O. Dukes - Fisiologia dos animais domésticos. 12ª ed. Rio de Janeiro: Guanabara Koogan, 2006. 926 p. 
TERRA JÚNIOR, J. A.; DANEZE, E. R.; TERRA, G. A.; ETCHEBEHERE, R. M.; BARBOSA, A. B. R.; TERRA, S. A. Subcostal schwannoma in pregnancy. Arquivos de Neuropsiquiatria, v. 70, n. 11, p. 901-902, 2012.

THEILEN, G. H.; MADEWELL, B. R. Tumors of the central nervous system and peripheral nerves. In: Veterinary cancer medicine. Philadelphia: Lea \& Febiger, 1979. p. 382-392.

WOODRUFF, J. M.; KOUREA, H. P.; LOIS, D. N.; SCHEITHAUER, B. W. Tumors of cranial and peripheral nerves. In: KLEIHUES, P.; CAVENEE, W. K. Pathology and genetics of tumors of the nervous system. Lyon: IARC, 2000. p. 164-174.

YAGER, J. A.; SCOTT, D. W. The skin and appendages. In: JUBB, K. V. F.; KENNEDY, P. C.; PALMER, N. Pathology of domestic animals. 4a ed. San Diego: Academic, 1993. p. 531-738.

YAGER, J. A.; WILCOCK, B. P. Color atlas and text of surgical pathology of the dog and cat: dermatopathology and skin tumors. London: Wolfe, 1994. p. 239-313.

ZACHARY, J. F. Sistema nervoso. In: MCGAVIN, M. D.; ZACHARY, J. F. Bases da patologia em veterinária. 4a ed. Rio de Janeiro: Elsevier, 2009. p. 833-972.

ZAMECNIK, M.; MICHAL, M. Perineurial cell differentiation in neurofibromas. report of eight cases including a case with composite perineuroma-neurofibroma features. Pathology

Research and Practice, v. 197, p. 537-544, 2001.

Autor para correspondência: Geórgia Modé Magalhães. Faculdade Dr. Francisco Maeda (FAFRAM/FE), Ituverava (SP). E-mail: georgiamode@hotmail.com 